Cite this: Analyst, 2014, 139, 3636

Received 3rd June 2013

Accepted 28th April 2014

DOI: 10.1039/c3an01104f

www.rsc.org/analyst

\title{
Design and development of heterologous competitive immunoassays for the determination of boscalid residues $\uparrow$
}

\author{
Francesc A. Esteve-Turrillas, ${ }^{a}$ Josep V. Mercader, ${ }^{a}$ Consuelo Agulló, ${ }^{b}$ Javier Marzo, ${ }^{b}$ \\ Antonio Abad-Somovilla ${ }^{\mathrm{b}}$ and Antonio Abad-Fuentes*a
}

Boscalid is a modern agrochemical belonging to the so-called chemical class of succinate dehydrogenase inhibitor fungicides. With the aim of developing rapid analytical screening methods for this relevant compound, we herein report the synthesis of new boscalid mimics and the study of their suitability for the production of polyclonal antibodies. Aliphatic spacer arms equivalent in length and composition were tethered at two different aromatic rings of the target molecular structure. These haptens, besides being used for immunization, were employed in the development of heterologous competitive enzymelinked immunosorbent assays (cELISAs) in order to improve assay detectability. Direct and indirect immunoassays were tailored and applied to the determination of samples with incurred boscalid residues. The assays were characterized in terms of sensitivity, specificity, trueness, and precision. Limit of quantification was established at $5 \mu \mathrm{g} \mathrm{kg}^{-1}$, coefficients of variation were lower than $20 \%$, and recoveries from spiked samples ranged from 90 to 137\%. Finally, ELISA performance was evaluated by Deming regression analysis with tomato and cucumber samples, selecting ultra-performance liquid chromatography-mass spectrometry as the reference method. The results showed that the proposed cELISAs are useful for the routine determination of boscalid fungicides in foods with high-sample throughput and affordable cost.

\section{Introduction}

Boscalid (Fig. 1) is a wide spectrum fungicide that acts by reducing fungi mitochondrial respiration through the inhibition of the enzyme succinate ubiquinone reductase (complex II) in the mitochondrial electron transport chain. ${ }^{1}$ This chemical is extensively employed for the management of several fungal diseases affecting fruits, vegetables, and ornamental crops. As a consequence, residues of this fungicide may remain in the final products; in fact, boscalid was the fourth most frequently encountered pesticide residue in food according to the latest European Union report. ${ }^{2}$ Recent studies also indicated that boscalid is a relevant contaminant in surface water and groundwater. ${ }^{3}$ Pesticide determination in food currently relies on the QuEChERS (Quick, Easy, Cheap, Effective, Rugged, and Safe) method, based on an extraction of the analyte with

${ }^{a}$ Institute of Agrochemistry and Food Technology, Consejo Superior de Investigaciones Científicas (IATA-CSIC), Agustí Escardino 7, 46980 Paterna, València, Spain. E-mail: aabad@iata.csic.es

${ }^{b}$ Department of Organic Chemistry, Universitat de València, Doctor Moliner 50, 46100 Burjassot, València, Spain. E-mail: antonio.abad@uv.es

$\dagger$ Electronic supplementary information (ESI) available: Additional chemicals and instruments, experimental details of hapten synthesis, and spectrometric characterization data for the synthesized compounds. See DOI: 10.1039/c3an01104f buffered acetonitrile followed by a clean-up of the extract with primary-secondary amine (PSA) and final chromatographic determination with spectrometry detection. ${ }^{4}$ The QuEChERS method has been employed for the extraction of boscalid residues in different food commodities before gas or liquid chromatography determination, including cereals, ${ }^{5}$ grapes, ${ }^{6}$ milk, ${ }^{7}$ paprika, ${ }^{8}$ or different fruits. ${ }^{9}$ Although chromatographic methods are sensitive and precise, they require high-cost equipment and are not well-suited to the analysis of large sample batches.

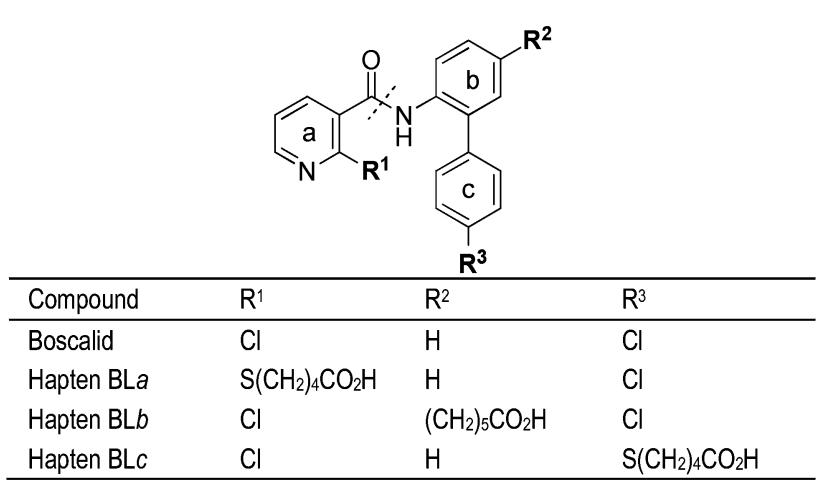

Fig. 1 Molecular structures of boscalid and the three synthesized haptens. 
The use of alternative methodologies such as immunochemical techniques is currently deemed as an excellent analytical option, in particular when a limited number of targets are sought. ${ }^{\mathbf{1 0 1 1}}$ The most extended immunochemical approach for the determination of low molecular weight analytes is based on competitive enzyme-linked immunosorbent assays (cELISAs) because this assay format is simple, affordable, and reliable. Additionally, cELISAs allow the sensitive and simultaneous analysis of numerous samples, commonly they require a simplified sample treatment, and allow on-site use. The first reported approach for the production of antibodies against boscalid was recently published by our group. ${ }^{12}$ In that previous study, immunoreagents were produced using hapten BLa (see Fig. 1), which contained a five-carbon atom spacer arm linked to the pyridine ring through a sulphur atom which replaced the chlorine atom present in the boscalid structure. It is well-known that antibody affinity and selectivity may be strongly influenced by the design of the immunizing hapten. ${ }^{13}$ Therefore, although previous antibodies derived from hapten BL $a$ displayed convenient analytical features, we wondered whether other possible tethering sites may give rise to antibodies of superior performance. Furthermore, the availability of different haptens and conjugates paves the way to develop heterologous cELISAs, a well-known approach to increase assay sensitivity in which the competitive reaction is driven by a protein conjugate carrying a hapten different to that employed in the immunogen. ${ }^{\mathbf{1 4 - 1 7}}$ In the present study, two novel haptens (BL $b$ and BLc , Fig. 1) with alternative linker attachment sites have been synthesized and employed in the preparation of protein conjugates and in the production of polyclonal antibodies to boscalid. A comprehensive evaluation of the generated immunoreagents has been carried out in order to develop cELISAs of high sensitivity. Finally, two new immunoassays, using direct and indirect cELISA formats, have been applied to the determination of boscalid residues in foods.

\section{Experimental}

\section{Reagents and instrumentation}

Boscalid (2-chloro- $N$-(4'-chlorobiphenyl-2-yl)nicotinamide, nicotinamide, $M_{\mathrm{w}} 343.2$, CAS registry no. 188425-85-6) and other fungicide standards were purchased from Fluka/Riedel-deHaën (Seelze, Germany) and Dr. Ehrenstorfer (Augsburg, Germany). Working solutions were prepared as concentrated solutions in $\mathrm{N}, \mathrm{N}$-dimethylformamide (DMF) and kept at $-20{ }^{\circ} \mathrm{C}$ in amber glass vials. Details about reagents, solvents, synthetic procedures, and techniques used in the structural characterization of synthesized compounds are described in the ESI. $\dagger$

Horseradish peroxidase (HRP), ovalbumin (OVA), o-phenylenediamine (OPD), and triphenyl phosphate (TPP) were purchased from Sigma-Aldrich (Madrid, Spain). Sephadex G-25 HiTrap Desalting columns from GE Healthcare (Uppsala, Sweden) were used for conjugate purification. Goat anti-rabbit immunoglobulin polyclonal antibody-peroxidase conjugate (GAR-HRP) was purchased from Biorad (Hercules, CA, USA). Bovine serum albumin (BSA) fraction $\mathrm{V}$ was purchased from Roche Applied Science (Mannheim, Germany). Foetal bovine serum (FBS) and Freund's adjuvants were purchased from Sigma-Aldrich (Madrid, Spain). Costar flat-bottom high-binding polystyrene ELISA plates were purchased from Corning (Corning, NY, USA). Ultraviolet-visible spectra and ELISA absorbances were read with a PowerWave HT from BioTek Instruments (Winooski, VT, USA). ELISA plates were washed with an ELx405 microplate washer also from BioTek Instruments. A T-25 ultraturrax blender and a Vortex mixer Ms2 were purchased from IKA (Staufen, Germany), and an Eppendorf 5804 centrifuge (Hamburg, Germany) was employed for the extraction of vegetables. PSA was obtained from Varian (Lake Forest, CA, USA).

The composition, concentration and $\mathrm{pH}$ of the employed buffers were as follows: (i) PB, $100 \mathrm{mM}$ sodium phosphate buffer ( $\mathrm{pH}$ 7.4); (ii) PBS, $10 \mathrm{mM}$ sodium phosphate buffer $(\mathrm{pH}$ 7.4) with $140 \mathrm{mM} \mathrm{NaCl}$; (iii) PBST, PBS containing $0.05 \%$ (v/v) Tween 20; (iv) CB, $50 \mathrm{mM}$ carbonate-bicarbonate buffer ( $\mathrm{pH}$ 9.6); (v) washing solution, $150 \mathrm{mM} \mathrm{NaCl}$ and $0.05 \%$ (v/v) Tween 20; (vi) enzyme substrate buffer, $25 \mathrm{mM}$ citrate and $62 \mathrm{mM}$ sodium phosphate buffer ( $\mathrm{pH} 5.4)$; and (vii) PBST $(2 \times), 20 \mathrm{mM}$ sodium phosphate buffer ( $\mathrm{pH} 7.4)$ with $280 \mathrm{mM} \mathrm{NaCl}$ and $0.05 \%(\mathrm{v} / \mathrm{v})$ Tween 20 .

\section{Hapten synthesis}

Haptens $\mathrm{BL} b$ and $\mathrm{BL} c$ used for the preparation of immunizing and assay conjugates were synthesized according to the synthetic routes detailed in Schemes 1 and 2, respectively. Full experimental details and physical and spectroscopic data of all the haptens and synthetic intermediates are provided in the ESI. $\dagger$

\section{Activation of haptens: preparation of the NHS-esters of BLb and BLc}

Carboxylated haptens were activated by the formation of the corresponding $N$-hydroxysuccinimidyl ester (NHS-ester) using $N, N^{\prime}$-disuccinimidyl carbonate (DSC) and $\mathrm{Et}_{3} \mathrm{~N}$ in dry acetonitrile as previously described. ${ }^{18}$ Hapten (1 eq.) and DSC (1.3-1.4 eq.) were dissolved in anhydrous acetonitrile $(1 \mathrm{~mL}$ per 0.1 mmol of hapten) under nitrogen in an ice-water bath. $\mathrm{Et}_{3} \mathrm{~N}(3.5$ eq.) was then added and the resulting mixture was stirred at room temperature until complete consumption of the starting material (as observed by thin layer chromatography using $\mathrm{CHCl}_{3}: \mathrm{MeOH} 9: 1$ as an eluent). The reaction mixture was diluted with $\mathrm{CHCl}_{3}$, washed with a $10 \%$ aqueous solution of $\mathrm{NaHCO}_{3}$ and brine, and dried over anhydrous $\mathrm{Na}_{2} \mathrm{SO}_{4}$. The residue obtained after evaporation of the solvent was purified by column chromatography, using $\mathrm{CHCl}_{3}$ as an eluent, affording the nearly pure NHS esters as determined by ${ }^{1} \mathrm{H}$ NMR spectra (copies of original spectra are included in the ESI $\dagger$ ).

BL $b$-NHS ester: (86\% yield). ${ }^{1} \mathrm{H}$-NMR $\left(300 \mathrm{MHz}, \mathrm{CDCl}_{3}\right) \delta$ (ppm) 8.71 (1H, d, $J=8.4 \mathrm{~Hz}, \mathrm{H}-5 \mathrm{PhPh}), 8.11$ (1H, s, NH), 7.81 $(1 \mathrm{H}, \mathrm{dd}, J=4.7,2.0 \mathrm{~Hz}, \mathrm{H}-6 \mathrm{Py}), 7.77(1 \mathrm{H}, \mathrm{dd}, J=7.7,2.0 \mathrm{~Hz}, \mathrm{H}-4$ Py), 7.11-6.85 (6H, m, H-2'/H-6 ${ }^{\prime}, \mathrm{H}-3^{\prime} / \mathrm{H}-5^{\prime}, \mathrm{H}-2$ and $\mathrm{H}-4 \mathrm{PhPh}$ ), $6.33(1 \mathrm{H}, \mathrm{dd}, J=7.7,4.7 \mathrm{~Hz}, \mathrm{H}-5 \mathrm{Py}), 2.36(2 \mathrm{H}, \mathrm{t}, J=7.6 \mathrm{~Hz}, \mathrm{H}-6)$, $2.15(2 \mathrm{H}, \mathrm{t}, J=7.3 \mathrm{~Hz}, \mathrm{H}-2), 1.77-1.51\left(4 \mathrm{H}, \mathrm{m}, \mathrm{COCH}_{2} \mathrm{CH}_{2} \mathrm{CO}\right)$, 1.45-1.28 (4H, m, H-3 and H-5), 1.22-1.08 (2H, m, H-4). 


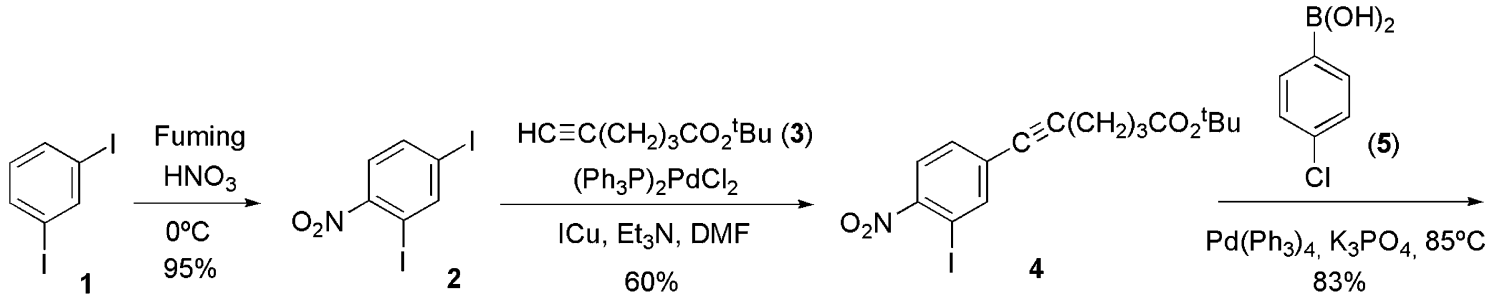<smiles>CCCCCCCC#Cc1ccc([N+](=O)[O-])c(-c2ccc(Cl)cc2)c1</smiles><smiles>CCOC(=O)OCc1ccc([N+](=O)[O-])c(-c2ccc(Cl)cc2)c1</smiles><smiles>CCCCCCc1ccc(N)c(-c2ccc(Cl)cc2)c1</smiles><smiles>CCCCCCCc1ccc(NC(=O)c2cccnc2Cl)c(-c2ccc(Cl)cc2)c1</smiles>

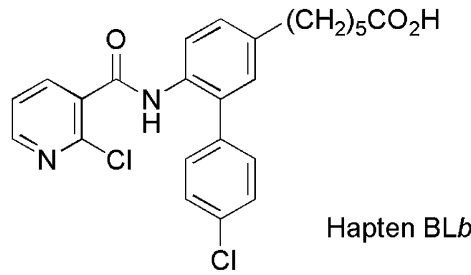

Scheme 1 Synthetic strategy for the preparation of hapten BLb.

BLc-NHS ester: (70\% yield). ${ }^{1} \mathrm{H}-\mathrm{NMR}\left(300 \mathrm{MHz}, \mathrm{C}_{6} \mathrm{D}_{6}\right) \delta$ (ppm) d 8.47-8.44 (2H, m, H-3' PhPh and H-6 Py), 8.22 (1H, br s, $\mathrm{NH}), 8.13$ (1H, dd, $J=7.7,1.9 \mathrm{~Hz}, \mathrm{H}-4 \mathrm{Py}), 7.48-7.42$ (8H, m, H-2/ $\mathrm{H}-6, \mathrm{H}-3 / \mathrm{H}-5, \mathrm{H}-4^{\prime}, \mathrm{H}-5^{\prime}, \mathrm{H}-6^{\prime} \mathrm{PhPh}$ and $\left.\mathrm{H}-5 \mathrm{Py}\right), 2.99$ (2H, t, $J=$ $7.0 \mathrm{~Hz}, \mathrm{H}-5), 2.83\left(4 \mathrm{H}\right.$, br s, $\left.\mathrm{COCH}_{2} \mathrm{CH}_{2} \mathrm{CO}\right), 2.66(2 \mathrm{H}, \mathrm{t}, J=7.2$ $\mathrm{Hz}, \mathrm{H}-2), 1.75-1.90(4 \mathrm{H}, \mathrm{m}, \mathrm{H}-3$ and $\mathrm{H}-4)$.

\section{Conjugation of activated haptens to proteins}

Purified activated haptens were coupled to proteins by the reaction of the NHS ester of the haptens with the free amine groups of the carrier, as previously reported for the preparation of BL $a$-type conjugates. ${ }^{\mathbf{1 2}}$ The two new haptens were conjugated to three proteins with different purposes: BSA was employed for

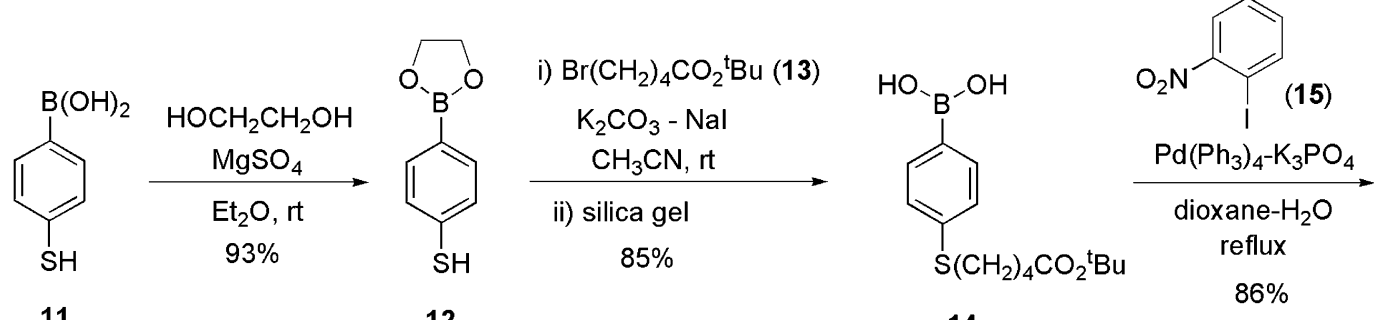

11

12

14<smiles>[R]c1ccccc1-c1ccc([SiH3])cc1</smiles>

16: $\mathrm{R}=\mathrm{NO}_{2} \quad \mathrm{Fe}, \mathrm{NH}_{4} \mathrm{Cl}$

17: $\mathrm{R}=\mathrm{NH}_{2} \curvearrowright$ EtOH- $\mathrm{H}_{2} \mathrm{O}$; reflux $90 \%$

Scheme 2 Synthetic strategy for the preparation of hapten BLC. 
immunogen preparation, HRP was used as an enzyme tracer in direct assays, and OVA as a coating conjugate in indirect assays. Protein-hapten conjugates were purified by gel filtration using $100 \mathrm{mM}$ phosphate buffer, $\mathrm{pH} 7.4$ as an eluent, and stored frozen at $-20{ }^{\circ} \mathrm{C}$. Coupling degrees were calculated at $280 \mathrm{~nm}$ from absorbance values from the protein before and after conjugation.

\section{Production of polyclonal antibodies}

Animal manipulation was carried out in compliance with Spanish laws and guidelines (RD1201/2005 and law 32/2007) and according to the European Directive 2010/63EU concerning the protection of animals used for scientific purposes. Each BSA conjugate was used to immunize two female New Zealand white rabbits. Animals were subcutaneously injected with $0.3 \mathrm{mg}$ of a BSA-hapten conjugate in $1 \mathrm{~mL}$ of a $1: 1$ mixture of $\mathrm{PB}$ and complete Freund's adjuvant. Animals were boosted at 21-day intervals with the same amount of immunogen suspended in a mixture of $0.5 \mathrm{~mL}$ of $\mathrm{PB}$ and $0.5 \mathrm{~mL}$ of incomplete Freund's adjuvant. Whole blood was collected by intracardiac puncture 10 days after the fourth injection. Blood samples were allowed to coagulate overnight at $4{ }^{\circ} \mathrm{C}$. Then, the serum was separated by centrifugation $(3000 \times g, 20 \mathrm{~min})$ and precipitated by adding 1 volume of a saturated aqueous solution of ammonium sulphate. This procedure was repeated again and the precipitates were finally stored at $4{ }^{\circ} \mathrm{C}$.

\section{Antibody-coated direct cELISAs}

Ninety-six-well polystyrene ELISA plates were coated with $100 \mu \mathrm{L}$ of a particular antiserum diluted in CB and plates were incubated overnight at room temperature. Coated plates were washed four times with washing solution and received, afterwards, $50 \mu \mathrm{L}$ per well of an analyte standard in PBS plus $50 \mu \mathrm{L}$ per well of an enzymatic tracer solution in PBST. The immunological reaction took place for $1 \mathrm{~h}$ at room temperature and then plates were washed as described above. Finally, a signal was generated by adding $100 \mu \mathrm{L}$ per well of a freshly prepared 2 $\mathrm{g} \mathrm{L}^{-1} o$-phenylenediamine solution containing $0.012 \%(\mathrm{v} / \mathrm{v})$ $\mathrm{H}_{2} \mathrm{O}_{2}$ in enzyme substrate buffer. The enzymatic reaction was stopped after $10 \mathrm{~min}$ at room temperature by addition of $100 \mu \mathrm{L}$ per well of $2.5 \mathrm{M}$ sulphuric acid. The absorbance was immediately read at $492 \mathrm{~nm}$ with a reference wavelength at $650 \mathrm{~nm}$.

\section{Conjugate-coated indirect cELISAs}

ELISA plates were coated with $100 \mu \mathrm{L}$ per well of coating conjugate solution in $\mathrm{CB}$ by overnight incubation at room temperature. Coated plates were washed as described above and then received $50 \mu \mathrm{L}$ per well of an analyte in PBS plus $50 \mu \mathrm{L}$ per well of an antiserum diluted in PBST. The immunological reaction took place for $1 \mathrm{~h}$ at room temperature and plates were washed again. Next, $100 \mu \mathrm{L}$ per well of a $10^{-4}$ dilution of a GARHRP conjugate in PBST containing 10\% FBS was added, and plates were incubated for $1 \mathrm{~h}$ at room temperature. After washing the plates, a signal was produced and the absorbance was read as aforementioned.

\section{Data processing}

Eight-point standard curves, including a blank, were prepared by six-fold serial dilution in PBS of a $100 \mu \mathrm{g} \mathrm{L} \mathrm{L}^{-1}$ boscalid solution. Experimental values were fitted to a four-parameter logistic equation using the SigmaPlot software package from SPSS Inc. (Chicago, IL, USA). Assay sensitivity was defined as the concentration of the analyte at the midpoint of the fitted curve, typically corresponding to a $50 \%$ inhibition $\left(\mathrm{IC}_{50}\right)$ of the maximum absorbance $\left(A_{\max }\right)$.

\section{Crop treatment and sample extraction}

Tomato and cucumber crops were in-field treated with Signum, a water dispersible granule formulation from BASF containing $26.7 \% \mathrm{w} / \mathrm{w}$ boscalid and $6.7 \% \mathrm{w} / \mathrm{w}$ pyraclostrobin. The commercial product was applied at the dose recommended by the manufacturer $(0.15 \% \mathrm{w} / \mathrm{w})$, and fruits were collected at different times (from 1 to 14 days) in order to obtain samples with different contents of boscalid. Samples were short-term stored at $4{ }^{\circ} \mathrm{C}$ upon reception. After all fruits had been harvested, samples were chopped, homogenized using an ultraturrax, and stored in polypropylene tubes at $-20{ }^{\circ} \mathrm{C}$ until analysis.

The QuEChERS method has been widely employed for the extraction and purification of fungicide residues from fruits and vegetables. ${ }^{4}$ In our case, $5 \mathrm{~mL}$ of chopped sample, plus $50 \mu \mathrm{L}$ internal standard (TPP at $50 \mathrm{mg} \mathrm{L}^{-1}$ ), were introduced in a 50 $\mathrm{mL}$ polypropylene centrifuge tube containing $0.5 \mathrm{~g}$ sodium acetate and $2 \mathrm{~g}$ anhydrous magnesium sulphate. Samples were then extracted with $5 \mathrm{~mL}$ of $1 \%(\mathrm{v} / \mathrm{v})$ acetic acid in acetonitrile by vortexing for $1 \mathrm{~min}$ and centrifuged for $5 \mathrm{~min}$ at $2200 \times \mathrm{g}$. Then, $1 \mathrm{~mL}$ extract was introduced into a $2 \mathrm{~mL}$ polypropylene centrifuge tube containing $50 \mathrm{mg}$ PSA and $150 \mathrm{mg}$ anhydrous magnesium sulphate, vortexed for $1 \mathrm{~min}$, and centrifuged for 5 $\min$ at $2200 \times g$. Finally, the purified extract was filtered through a $0.22 \mu \mathrm{m}$ Teflon filter and analyzed by the proposed direct and indirect cELISAs, as well as by the reference chromatographic method.

\section{Chromatographic analysis}

Ultra performance liquid chromatography (UPLC) coupled to a triple quadrupole mass spectrometer was selected as the reference methodology in order to obtain maximum sensitivity and selectivity in the analysis of boscalid residues. UPLC was performed on a Waters Acquity UPLC system from Waters (Milford, MA, USA), equipped with a binary solvent delivery system, an autosampler, and a $\mathrm{BEH} \mathrm{C}_{18}(1.7 \mu \mathrm{m}, 2.1 \times 50 \mathrm{~mm})$ column. The injection volume was set at $5 \mu \mathrm{L}$ and the mobile phase consisted of (A) $0.5 \%(\mathrm{v} / \mathrm{v})$ formic acid in water and (B) acetonitrile. The gradient started at $30 \%$ of $\mathrm{B}$, was linearly increased to $85 \%$ in $3.4 \mathrm{~min}$, and then to $98 \%$ in $1.7 \mathrm{~min}$ at a flow rate of $350 \mu \mathrm{L}$ $\min ^{-1}$. The obtained retention times under the aforementioned conditions were 3.57 and $4.25 \mathrm{~min}$ for boscalid and TPP, respectively. Tandem mass acquisitions were performed in a Waters Acquity triple quadrupole mass spectrometry detector, equipped with a Z-spray electrospray ionization source, with 3.5 
$\mathrm{kV}$ capillary voltage, $120{ }^{\circ} \mathrm{C}$ source temperature and $300{ }^{\circ} \mathrm{C}$ desolvation temperature. The employed parameters were: ESI+, parent ion $344.2 \mathrm{~m} / \mathrm{z}$, daughter ions 139.9 and $307.8 \mathrm{~m} / \mathrm{z}, 20 \mathrm{eV}$ collision energy and $35 \mathrm{~V}$ cone energy for boscalid; and ESI+, parent ion $328.3 \mathrm{~m} / \mathrm{z}$, daughter ions 77.0 and $152.4 \mathrm{~m} / \mathrm{z}, 30 \mathrm{eV}$ collision energy and $25 \mathrm{~V}$ cone energy for TPP.

\section{Results and discussion}

\section{Synthesis of boscalid haptens BLb and BLc}

The structure of boscalid consists of three interconnected aromatic rings, thus providing multiple potential sites for the introduction of a functionalized linker, and therefore for hapten synthesis. Our group recently reported the synthesis and antibody-eliciting properties of hapten BLa, which bears a carboxylate aliphatic spacer arm at the chloropyridyl ring of the boscalid framework. This synthetic strategy was chosen because substitution of a chlorine atom by a sulfur atom has been demonstrated as a convenient approach able to deliver suitable mimics of the target analyte..$^{13,19-22}$ Although high-affinity antibodies were obtained through that hapten, we thought there may be still room for improvement, so alternative tethering sites were deemed. Computer-assisted molecular modeling did not provide conclusive clues upon the optimum hapten structure for anti-boscalid antibody generation, so we finally decided to address the preparation of boscalid mimics through the two other aromatic rings that constitute the whole molecule. Overall, the synthesis of so-called haptens $\mathrm{BL} b$ and $\mathrm{BL} c$ followed a common strategy for the construction of the hapten framework based on the initial preparation of conveniently functionalized nicotinoyl and 2-aminobiphenyl synthons (rings a and b/c, respectively, Fig. 1), which are coupled together to form the amide bond.

Following this strategy, the synthesis of hapten $\mathrm{BL} b$ required the preparation of the synthon corresponding to the biphenyl moiety, compound 8. As outlined in Scheme 1, the synthesis of compound 8 started from 2,4-diiodo-1-nitrobenzene (2), easily available from 1,3-diiodobenzene (1), ${ }^{23}$ and is based on the different reactivity of the two iodinated positions against palladium catalyzed cross-coupling processes. First, the spacer arm was introduced at the appropriate position of the aromatic ring subunit by means of a regioselective Sonogashira coupling with tert-butyl hex-5-ynoate (3), which was followed by a SuzukiMiyaura cross-coupling reaction with 4-chlorophenylboronic acid (5) to form the biphenyl derivative 6 . Hydrogenation of the acetylenic triple bond using Wilkinson's catalyst followed by reduction of the nitro group with iron completed the preparation of the biphenyl amine $\mathbf{8}$, from which the synthesis of hapten $\mathrm{BL} b$ was readily accomplished via coupling with 2chloronicotinoyl chloride (9) and subsequent acid-catalyzed hydrolysis of the tert-butyl ester moiety.

The synthesis of hapten BLc required the preparation of the 2-aminobiphenyl intermediate 17 (Scheme 2). Its synthesis started from 4-mercaptophenylboronic acid (11) that was initially transformed into the corresponding ethylene glycol ester 12 in order to avoid interference of the boronic acid moiety in the subsequent alkylation reaction of the mercaptide group.
Further alkylation of $\mathbf{1 2}$ with tert-butyl 5-bromopentanoate (13) followed by chromatography on a silica gel, which induced the hydrolysis of the ethylene glycol ester moiety, led to the formation of boronic acid 14. Completion of the synthesis of 17 was efficiently accomplished by means of a Suzuki-Miyaura cross-coupling reaction followed by reduction of the nitro group. The last steps for the preparation of hapten BLc were straightforward, involving the formation of the amide linkage by the reaction between $\mathbf{1 7}$ and 2-chloronicotinoyl chloride (9) followed by formic acid catalyzed hydrolysis of the tert-butyl ester moiety.

\section{Characterization of immunoreagents}

Four antisera, named $\mathrm{BL} b \# 1, \mathrm{BL} b \# 2, \mathrm{BL} c \# 1$, and $\mathrm{BL} c \# 2$, were obtained from the two novel immunogen conjugates BSA-BL $b$ and BSA-BLc. Polyclonal antibodies BLa\#1 and BLa\#2, and the respective protein conjugates previously produced with hapten BL $a$, were also incorporated in this study. ${ }^{12}$ A checkerboard competitive screening analysis was employed to characterize the performance of the immunoreagents in both direct and indirect cELISAs.

For direct cELISA, plates were coated with $1 / 6 \times 10^{4}$ and $1 / 3$ $\times 10^{4}$ dilutions of the antiserum, and the next day, a range of enzyme tracer concentrations (from 1 to $300 \mu \mathrm{g} \mathrm{L}^{-1}$ ) was evaluated under competitive conditions. For heterologous combinations lower antiserum dilutions $\left(1 / 3 \times 10^{3}\right.$ and $\left.1 / 10^{4}\right)$ were used because of the poorer recognition of heterologous conjugates. A collection of inhibition curves was so obtained for each pair of immunoreagents, and a summary of the parameters ( $A_{\max }$, slope, and $\mathrm{IC}_{50}$ values) of the inhibition curves resulting from the more adequate concentrations is shown in Table 1. Homologous assays showed $\mathrm{IC}_{50}$ values in the $0.4-1.6 \mu \mathrm{g} \mathrm{L}^{-1}$ range, depending on the employed antisera. The use of heterologous conjugates improved the sensitivity of the assays, as illustrated by mixed combinations of $\mathrm{BL} a$ - and $\mathrm{BL} b$-derived immunoreagents. However, in the case of antibodies BLc\#1 and $\mathrm{BL} c \# 2, \mathrm{IC}_{50}$ values hardly improved by using tracers HRP-BL $a$ or HRP-BL $b$. Finally, the assay with antiserum BL $b \# 2$ and tracer HRP-BL $a$ brought about the most sensitive direct assay $\left(\mathrm{IC}_{50}=\right.$ $\left.0.2 \mu \mathrm{g} \mathrm{L}^{-1}\right)$.

For indirect cELISA, plates were coated with 0.01 and $0.1 \mathrm{mg}$ $\mathrm{L}^{-1}$ OVA conjugate solutions, and the competitive step was carried out using a range of antisera dilutions (from $1 / 10^{4}$ to 1 / $10^{6}$ ). Indirect assays also showed a low recognition of heterologous conjugates, so a higher coating concentration was assessed $\left(1.0 \mathrm{mg} \mathrm{L}^{-1}\right)$. Table 1 shows the main parameters from the inhibition curves of the more sensitive combinations. IC $_{50}$ values obtained for homologous assays ranged from 0.3 to 1.7 $\mu \mathrm{g} \mathrm{L}^{-1}$, and sensitivity improvements were again noticed by using heterologous conjugates. Antiserum BL $b \# 2$ in combination with the coating conjugate OVA-BL $a$ resulted in an assay of remarkable sensitivity $\left(\mathrm{IC}_{50}=0.1 \mu \mathrm{g} \mathrm{L}^{-1}\right)$.

\section{Selectivity}

Cross-reactivity studies were performed in order to find whether other related compounds could be recognized by the proposed 
Table 1 Inhibition curve parameters obtained from the competitive bidimensional characterization of antisera in direct and indirect cELISA formats $(n=3)^{a}$

\begin{tabular}{|c|c|c|c|c|c|c|c|c|c|c|c|c|c|c|}
\hline Antibody & $\begin{array}{l}\text { Antibody } \\
\text { dilution }\end{array}$ & \multicolumn{3}{|c|}{$\begin{array}{l}\text { HRP tracer } \\
\left(\mu \mathrm{g} \mathrm{L}^{-1}\right)\end{array}$} & $A_{\max }$ & Slope & $\begin{array}{l}\mathrm{IC}_{50} \\
\left(\mu \mathrm{g} \mathrm{L}^{-1}\right)\end{array}$ & $\begin{array}{l}\text { Antibody } \\
\text { dilution }\end{array}$ & \multicolumn{3}{|c|}{$\begin{array}{l}\text { OVA coating } \\
\left(\mathrm{mg} \mathrm{L}^{-1}\right)\end{array}$} & $A_{\max }$ & Slope & $\begin{array}{l}\mathrm{IC}_{50} \\
\left(\mu \mathrm{g} \mathrm{L}^{-1}\right)\end{array}$ \\
\hline BLa\#1 & $6 \times 10^{4}$ & 3 & - & - & 0.79 & 0.52 & 0.4 & $3 \times 10^{5}$ & 0.1 & - & - & 1.05 & 0.67 & 0.9 \\
\hline \multirow[t]{3}{*}{ BLa\#2 } & $6 \times 10^{4}$ & 3 & - & - & 1.00 & 0.61 & 1.6 & $10^{6}$ & 0.1 & - & - & 0.97 & 0.55 & 0.9 \\
\hline & $10^{4}$ & - & 10 & - & 1.50 & 1.48 & 0.9 & $10^{5}$ & - & 1 & - & 0.96 & 0.52 & 0.5 \\
\hline & $10^{4}$ & - & - & 10 & 1.39 & 0.84 & 1.7 & $3 \times 10^{5}$ & - & - & 0.1 & 0.78 & 0.71 & 1.7 \\
\hline \multirow[t]{3}{*}{ BL $b \# 1$} & $10^{4}$ & 30 & - & - & 0.96 & 0.96 & 0.5 & $3 \times 10^{4}$ & 0.1 & - & - & 0.73 & 1.07 & 0.4 \\
\hline & $6 \times 10^{4}$ & - & 3 & - & 0.73 & 0.66 & 0.6 & $3 \times 10^{5}$ & - & 0.1 & - & 1.11 & 0.62 & 0.3 \\
\hline & $10^{4}$ & - & - & 10 & 0.92 & 0.78 & 0.9 & $10^{5}$ & - & - & 0.1 & 0.71 & 0.65 & 0.3 \\
\hline & $10^{4}$ & - & 10 & - & 1.20 & 1.05 & 1.6 & $3 \times 10^{4}$ & - & 0.1 & - & 0.76 & 0.76 & 0.8 \\
\hline & $6 \times 10^{4}$ & - & - & 3 & 1.17 & 0.54 & 0.8 & $10^{5}$ & - & - & 0.01 & 1.36 & 0.76 & 0.9 \\
\hline \multirow[t]{3}{*}{$\mathrm{BL} c \# 2$} & $3 \times 10^{3}$ & 10 & - & - & 0.90 & 0.68 & 1.9 & $3 \times 10^{4}$ & 0.1 & - & - & 1.05 & 0.70 & 5.1 \\
\hline & $10^{4}$ & - & 100 & - & 0.77 & 0.75 & 6.7 & $10^{4}$ & - & 0.1 & - & 0.81 & 1.12 & 1.1 \\
\hline & $6 \times 10^{4}$ & - & - & 3 & 1.25 & 0.76 & 1.6 & $10^{5}$ & - & - & 0.01 & 1.14 & 0.76 & 1.7 \\
\hline
\end{tabular}

${ }^{a}$ For each combination of immunoreagents, the particular concentrations in the table are those resulting in the lowest $\mathrm{IC}_{50}$ value provided that $A_{\text {max }}$ was higher than 0.6. IC $_{50}$ values from homologous combinations are indicated in bold.

assays. Accordingly, 22 widely used fungicides (pyraclostrobin, kresoxim-methyl, trifloxystrobin, azoxystrobin, dimoxystrobin, fluoxastrobin, metominostrobin, picoxystrobin, fenhexamid, captan, mepanipyrim, pyrimethanil, procymidone, tolylfluanid, cyazofamid, tebuconazole, fenamidone, fludioxonil, vinclozolin, imidacloprid, cyprodinil, and benzanilide) were assayed as competitors. Serial dilutions in PBS of each fungicide from a 10 $\mu \mathrm{M}$ standard solution were prepared and assayed by the direct and indirect assays in a homologous format. No inhibition was identified by any of these agrochemicals, so it could be concluded that all the produced antisera were highly specific to boscalid.

\section{Proposed assays}

Standard inhibition curve conditions and parameters of the most sensitive direct and indirect immunoassays (antiserum BL $b \# 2$ with BLa-derived assay conjugates) are shown in Table 2. Final immunoreagent concentrations for each immunoassay were slightly increased with respect to those in Table 1 in order to obtain higher $A_{\max }$ values. As it can be seen in Table 2, both assays were nearly equivalent in terms of detectability.

\section{Solvent tolerance}

The presence of organic solvents in the test samples may influence the interaction among the different reagents involved in the competitive step of the assay, thus causing inadequate quantifications of the target analyte. Solvent sources may come from the sample itself, like ethanol in alcoholic drinks or
Table 2 Assay conditions and analytical parameters of the optimized immunoassays $^{a}$

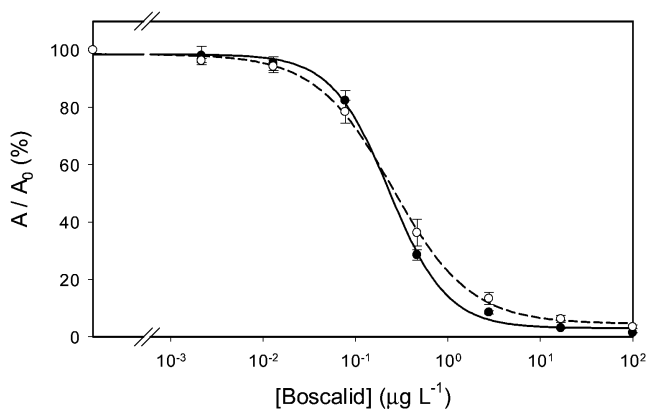

\begin{tabular}{lll}
\hline & \multicolumn{1}{c}{ cELISA } \\
\cline { 2 - 3 } Format & Direct $(\bullet)$ & Indirect $(\bigcirc)$ \\
\hline Antibody & BL $b \# 2\left(1 / 3 \times 10^{3}\right)$ & BL $b \# 2\left(1 / 2 \times 10^{4}\right)$ \\
Conjugate & HRP-BL $a\left(40 \mu \mathrm{g} \mathrm{L}^{-1}\right)$ & OVA-BL $a\left(100 \mu \mathrm{g} \mathrm{L}^{-1}\right)$ \\
$A_{\max }$ & $1.73 \pm 0.09$ & $1.14 \pm 0.18$ \\
$A_{\min }$ & $0.051 \pm 0.007$ & $0.055 \pm 0.016$ \\
Slope & $-1.34 \pm 0.11$ & $-1.04 \pm 0.03$ \\
$\operatorname{IC}_{50}\left(\mu \mathrm{g} \mathrm{L}^{-1}\right)$ & $0.22 \pm 0.02$ & $0.26 \pm 0.05$ \\
$\operatorname{LOD}^{b}\left(\mu \mathrm{g} \mathrm{L}^{-1}\right)$ & 0.05 & 0.03
\end{tabular}

${ }^{a}$ Values are the mean of 8 independent experiments. ${ }^{b}$ LOD was estimated as the boscalid concentration causing a $10 \%$ inhibition of $A_{\max }$. 

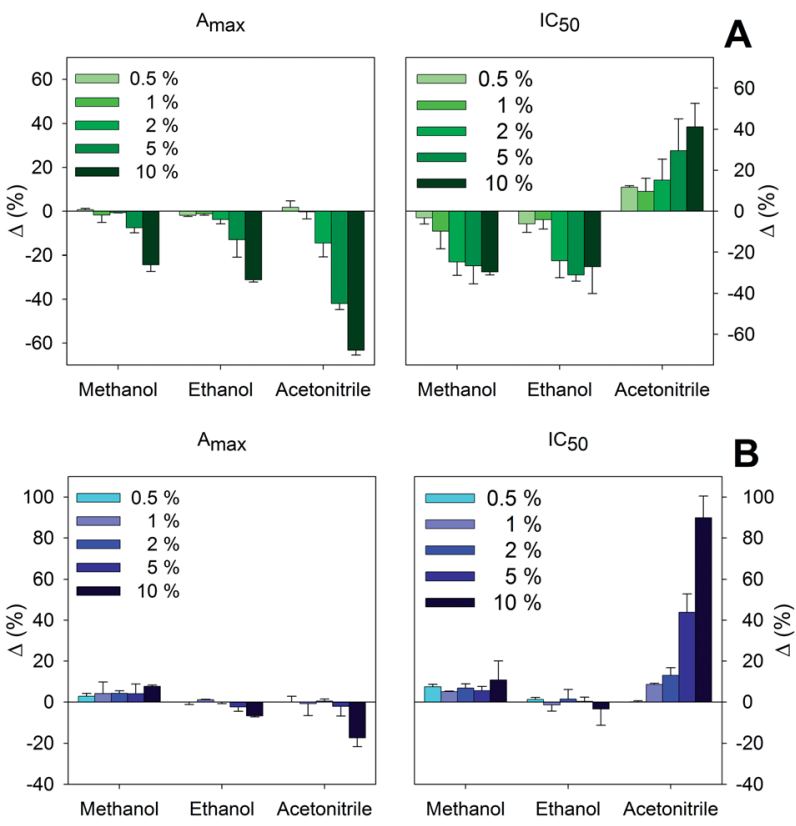

Fig. 2 Variation of $A_{\max }$ and $I C_{50}$ values of the proposed direct (A) and indirect (B) CELISAs as a function of the presence of organic solvents.

fermented samples, or they may have been intentionally added, such as acetonitrile and methanol, two common solvents that are usually employed in the extraction process. Fig. 2 shows the effect of different percentages of methanol, ethanol, and acetonitrile over the main parameters of the calibration curves of the proposed immunoassays. In general, $A_{\max }$ was less affected by the presence of solvents than $\mathrm{IC}_{50}$ values, and any of the assayed solvents could be employed up to $5 \%$ with acceptable variations, lower than $20 \%$. However, modifications in $\mathrm{IC}_{50}$ values were more noticeable - solvent contents higher than $2 \%$ appreciably altered the assay sensitivity. Accordingly, samples should be adequately diluted in assay buffer in order to obtain accurate results.

\section{Determination of boscalid in vegetables}

Tomato and cucumber crops were selected as model commodities to evaluate the performance of the developed immunoassays. Firstly, matrix effects were evaluated using boscalid-free tomato and cucumber samples (as measured by UPLC-MS-MS) that were extracted by QuEChERS. Extracts were diluted with deionized water (5-, 25-, 50-, 250- and 500-fold) and these solutions were used to prepare boscalid standard curves. After running the corresponding competitive assays (data not shown), it was evidenced that inhibition curves equivalent to those carried out in buffer were attained with extracts diluted at least 50 -fold, so this was the minimum sample dilution required in order to obtain accurate results.

Secondly, recovery studies were performed using analyte-free samples spiked with boscalid at different concentrations $(5,10$, $50,100,500,1000$, and $5000 \mu \mathrm{g} \mathrm{kg}^{-1}$ ). Fortified samples were homogenized by vortex mixing, extracted by QuEChERS, and measured by the proposed cELISAs after being diluted 1/100
Table 3 Recoveries of tomato and cucumber spiked samples determined by direct and indirect cELISAs based on antibody BLb\#2 and BLa-derived conjugates $^{a}$

\begin{tabular}{llcc}
\hline & & \multicolumn{2}{c}{ Recovery $(\% \pm \mathrm{s}, n=5)$} \\
\cline { 3 - 4 } Assay format & {$[\text { Boscalid }]^{b}\left(\mu \mathrm{g} \mathrm{kg}^{-1}\right)$} & Tomato & Cucumber \\
\hline \multirow{2}{*}{ Direct } & 5 & $128 \pm 9$ & $137 \pm 27$ \\
& 10 & $113 \pm 17$ & $113 \pm 8$ \\
& 50 & $99 \pm 7$ & $102 \pm 7$ \\
& 100 & $97 \pm 7$ & $99 \pm 8$ \\
Indirect & 500 & $91 \pm 15$ & $92 \pm 14$ \\
& 1000 & $94 \pm 14$ & $97 \pm 4$ \\
& 5000 & $96 \pm 7$ & $90 \pm 5$ \\
& 5 & $97 \pm 8$ & $103 \pm 8$ \\
& 10 & $108 \pm 5$ & $98 \pm 10$ \\
& 50 & $96 \pm 8$ & $94 \pm 12$ \\
& 100 & $99 \pm 3$ & $101 \pm 15$ \\
& 500 & $96 \pm 11$ & $102 \pm 6$ \\
& 1000 & $99 \pm 10$ & $97 \pm 9$ \\
& 5000 & $97 \pm 3$ & $107 \pm 18$
\end{tabular}

${ }^{a}$ Samples spiked at $5-50 \mu \mathrm{gg}^{-1}$ were diluted in assay buffer $1 / 100$, whereas samples spiked at $100-500 \mu \mathrm{g} \mathrm{kg}^{-1}$ were diluted $1 / 1000$. ${ }^{b}$ Boscalid concentration in $\mu \mathrm{g} \mathrm{kg}{ }^{-1}$.

(low-level spiked samples) and 1/1000 (high-level spiked samples) with buffer. As shown in Table 3, adequate recovery values were obtained for all the evaluated concentrations. Concerning precision, relative standard deviation (RSD) values were lower than $20 \%$. Accordingly, the limit of quantification

Table 4 Determination of boscalid residues in tomato (T) and cucumber (C) samples by UPLC-MS-MS and heterologous CELISAs

\begin{tabular}{llccc}
\hline \multirow{4}{*}{ Sample } & Name & UPLC-MS-MS & Direct cELISA & Indirect cELISA \\
\cline { 3 - 5 } In-field & T1 & $510 \pm 50$ & $440 \pm 30$ & $490 \pm 40$ \\
treated & T2 & $420 \pm 50$ & $420 \pm 40$ & $420 \pm 60$ \\
& T3 & $230 \pm 20$ & $230 \pm 20$ & $230 \pm 30$ \\
& T4 & $260 \pm 20$ & $235 \pm 8$ & $240 \pm 10$ \\
& C1 & $250 \pm 40$ & $350 \pm 20$ & $350 \pm 60$ \\
& C2 & $460 \pm 30$ & $400 \pm 30$ & $440 \pm 30$ \\
& C3 & $230 \pm 30$ & $450 \pm 20$ & $490 \pm 30$ \\
Blind & C4 & $260 \pm 40$ & $430 \pm 30$ & $450 \pm 50$ \\
spiked & T5 & $150 \pm 20$ & $140 \pm 10$ & $130 \pm 20$ \\
& T6 & $270 \pm 30$ & $260 \pm 10$ & $260 \pm 10$ \\
& T7 & $400 \pm 30$ & $390 \pm 10$ & $400 \pm 20$ \\
& T8 & $870 \pm 90$ & $780 \pm 30$ & $760 \pm 20$ \\
& T9 & $1400 \pm 100$ & $1270 \pm 60$ & $1250 \pm 40$ \\
& T10 & $1600 \pm 200$ & $1570 \pm 30$ & $1500 \pm 100$ \\
& T11 & $1900 \pm 200$ & $1810 \pm 90$ & $1860 \pm 80$ \\
& T12 & $2400 \pm 200$ & $2090 \pm 80$ & $2500 \pm 100$ \\
& C5 & $210 \pm 10$ & $200 \pm 20$ & $210 \pm 10$ \\
& C6 & $310 \pm 30$ & $290 \pm 20$ & $300 \pm 10$ \\
& C7 & $600 \pm 80$ & $440 \pm 20$ & $550 \pm 40$ \\
& C8 & $960 \pm 90$ & $940 \pm 80$ & $940 \pm 80$ \\
C9 & $1300 \pm 100$ & $1300 \pm 70$ & $1300 \pm 100$ \\
& C10 & $1900 \pm 200$ & $1700 \pm 100$ & $1790 \pm 50$ \\
& C11 & $2000 \pm 200$ & $1700 \pm 100$ & $1870 \pm 20$ \\
& C12 & $2600 \pm 200$ & $2100 \pm 100$ & $2560 \pm 30$ \\
& & & &
\end{tabular}


(LOQ), defined as the minimal concentration that can be measured with satisfactory accuracy (recoveries between 80 and $120 \%$ ) and precision (RSD lower than 20\%), was established at 5 $\mu \mathrm{g} \mathrm{kg}{ }^{-1}$ for the indirect assay and at $10 \mu \mathrm{g} \mathrm{kg}{ }^{-1}$ for the direct assay.

Finally, samples from in-field treated crops were analysed by the developed immunochemical methods and by UPLC-MS-MS as a reference procedure. Table 4 shows that boscalid concentrations in these samples ranged from 230 to $510 \mu \mathrm{g} \mathrm{kg}^{-1}$, wellbelow the EU maximum residue limit for cucumbers and tomatoes $\left(3 \mathrm{mg} \mathrm{kg}^{-1}\right)$. In order to get a larger number of positive samples covering a wide range of boscalid concentrations, blind spiked samples were prepared enabling a more comprehensive validation of the immunoassays. A total of 16 samples were fortified by an external operator and analysed by the three analytical methodologies, the two proposed cELISAs and UPLCMS-MS (Table 4). Boscalid concentrations ranged from 150 to $2600 \mu \mathrm{g} \mathrm{kg}^{-1}$. Deming regression analysis was employed for data comparison, and Fig. 3 shows the obtained regression graphs and the 95\% confidence interval (CI) bands for direct and indirect cELISAs versus UPLC-MS-MS.

Comparison of the direct assay with UPLC-MS-MS produced a Deming regression slope of 0.93 (CI from 0.85 to 1.01) and an
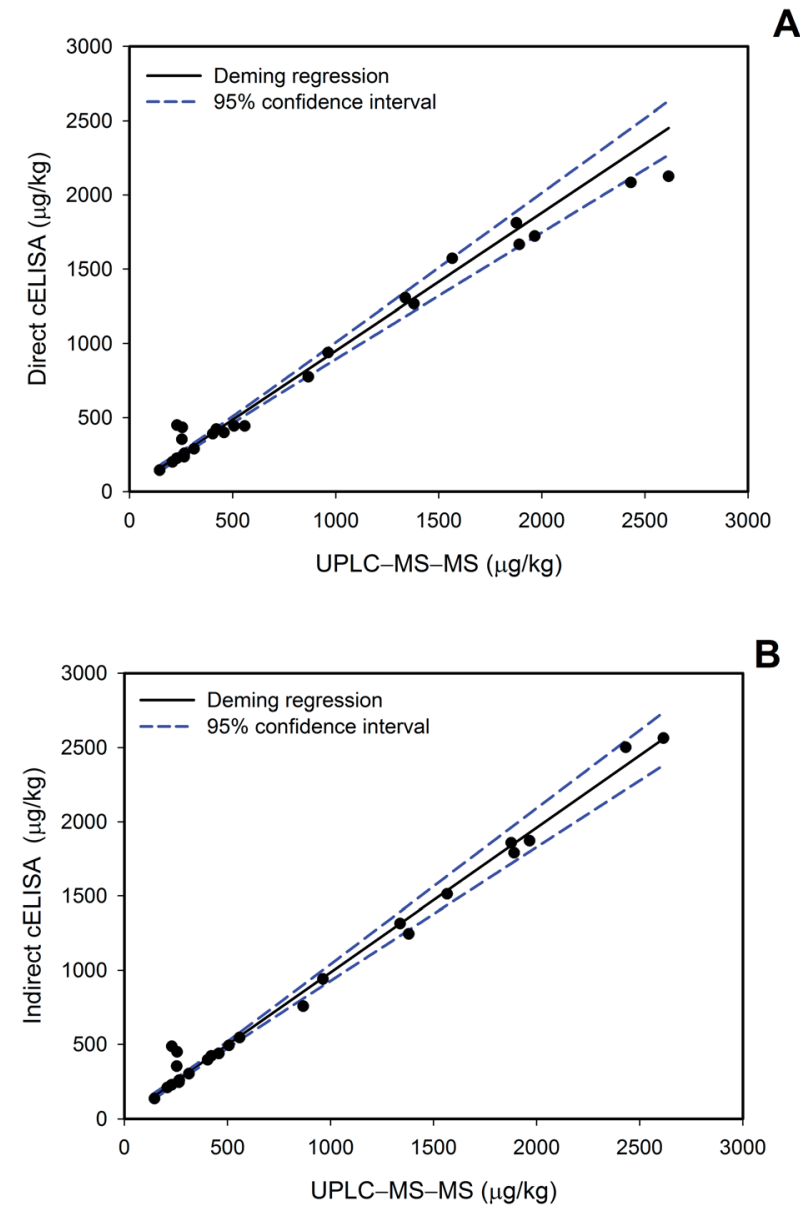

Fig. 3 Deming regression analysis of $\operatorname{direct}(\mathrm{A})$ and indirect $(\mathrm{B}) \mathrm{CELISAs}$ vs. UPLC-MS-MS with tomato and cucumber samples. intercept of 21 (CI from -9 to 52); and for the indirect assay the slope was 0.98 (CI from 0.90 to 1.05) and the intercept was 9 (CI from -20 to 38 ). Therefore, both Deming fittings have slopes and intercepts statistically equal to 1 and 0 , respectively; it could therefore be concluded that the developed cELISAs provided results statistically comparable to the reference method.

\section{Conclusions}

With the aim of producing immunoreagents enabling the sensitive analysis of boscalid, novel polyclonal antibodies have been produced against this modern fungicide using two original hapten molecules carrying a spacer arm tethered to alternative positions of the target molecule. After coupling to carrier proteins, the antibody-eliciting properties of these two haptens (BL $b$ and $\mathrm{BL} c$ ) were assessed and compared with that of a previously synthesized hapten (BLa) that had been derivatized at a different aromatic ring. Remarkably, our results evidenced that all three haptens brought about antibodies of similar affinity to boscalid, so it could be concluded that the three aromatic rings of the boscalid framework are in closely equivalent positions in order to generate convenient binders. Most importantly, the availability of such a wide collection of antibodies and conjugates allowed us to set up a number of different combinations of immunoreagents that eventually resulted in competitive heterologous immunoassays displaying superior performance. Thus, the combination of antiserum $\mathrm{BL} b \# 2$ with $\mathrm{BL} a$-type assay conjugates gave rise to assays that improved 4-6 times the $\mathrm{IC}_{50}$ values of the homologous arrangements. The two most sensitive immunoassays in the direct and indirect formats (LOD 0.03-0.05 $\mu \mathrm{g} \mathrm{L}^{-1}$ ) were applied to the analysis of fungicide residues in cucumbers and tomatoes. Upon validation, the proposed assays showed a LOQ of 5$10 \mu \mathrm{g} \mathrm{kg}^{-1}$, and results statistically equivalent to those of a chromatographic reference procedure were obtained.

\section{Acknowledgements}

This work was supported by Ministerio de Educación y Ciencia (AGL2006-12750-C02-01/02/ALI and AGL2009-12940-C02-01/02/ ALI) and cofinanced by FEDER funds. F.A.E.T. and J.V.M. were hired by CSIC with postdoctoral contracts, under the JAE and the Ramón y Cajal programs, respectively. All contracts were cofinanced by Ministerio de Ciencia e Innovación and by the European Social Fund. We also thank Ana Izquierdo-Gil and Laura López-Sánchez for excellent technical assistance. Limited amounts of the described immunoreagents are available upon request.

\section{Notes and references}

1 H. F. Avenot and T. J. Michailides, Plant Dis., 2007, 91, 1345. 2 European Food Safety Authority, EFSA J., 2013, 11, 3130.

3 T. J. Reilly, K. L. Smalling, J. L. Orlando and K. M. Kuivila, Chemosphere, 2012, 89, 228.

4 S. J. Lehotay, J. AOAC Int., 2007, 90, 485.

5 S. Walorczyk, J. Chromatogr. A, 2008, 1208, 202. 
6 M. Corrales, A. Fernandez, M. G. V. Pinto, P. Butz, C. M. A. P. Franz, E. Schuele and B. Tauscher, Food Chem. Toxicol., 2010, 48, 3471.

7 E. Tsiplakou, C. J. Anagnostopoulos, K. Liapis, S. A. Haroutounian and G. Zervas, Chemosphere, 2010, 80, 504.

8 S. W. Lee, J. H. Choi, S. K. Cho, H. A. Yu, A. M. Abd El-Aty and J. H. Shim, J. Chromatogr. A, 2011, 1218, 4366.

9 S. Walorczyk, J. Chromatogr. A, 2012, $1222,98$.

10 V. S. Morozova, A. I. Levashova and S. A. Eremin, J. Anal. Chem., 2005, 60, 202.

11 Z. L. Xu, Y. D. Shen, R. C. Beier, J. Y. Yang, H. T. Lei, H. Wang and Y. M. Sun, Anal. Chim. Acta, 2009, 647, 125.

12 A. Abad-Fuentes, F. A. Esteve-Turrillas, C. Agulló, A. AbadSomovilla and J. V. Mercader, Food Chem., 2012, 135, 276.

13 C. Suarez-Pantaleon, J. V. Mercader, C. Agullo, A. AbadSomovilla and A. Abad-Fuentes, Org. Biomol. Chem., 2011, 9, 4863.

14 K. C. Ahn, S. J. Gee, H. J. Tsai, D. Bennett, M. G. Nishioka, A. Blum, E. Fishman and B. D. Hammock, Environ. Sci. Technol., 2009, 43, 7784 .
15 J. Ramon-Azcon, F. Sanchez-Baeza, N. Sanvicens and M. P. Marco, J. Agric. Food Chem., 2009, 57, 375.

16 J. V. Mercader, J. Parra, F. A. Esteve-Turrillas, C. Agulló, A. Abad-Somovilla and A. Abad-Fuentes, Food Control, 2012, 26, 162.

17 J. Parra, J. V. Mercader, C. Agullo, A. Abad-Somovilla and A. Abad-Fuentes, Anal. Chim. Acta, 2012, 715, 105.

18 F. A. Esteve-Turrillas, J. Parra, A. Abad-Fuentes, C. Agullo, A. Abad-Somovilla and J. V. Mercader, Anal. Chim. Acta, 2010, 682, 93.

19 R. O. Harrison, M. H. Goodrow and B. D. Hammock, J. Agric. Food Chem., 1991, 39, 122.

20 P. C. C. Feng, S. R. Horton and C. R. Sharp, J. Agric. Food Chem., 1992, 40, 211.

21 J. J. Manclus, J. Primo and A. Montoya, J. Agric. Food Chem., 1996, 44, 4052.

22 J. V. Mercader, C. Agullo, A. Abad-Somovilla and A. AbadFuentes, Org. Biomol. Chem., 2011, 9, 1443.

23 Z. H. Zhang, Z. G. Zha, C. S. Gan, C. F. Pan, Y. Q. Zhou, Z. Y. Wang and M. M. Zhou, J. Org. Chem., 2006, 71, 4339. 